\title{
Effet de densité initiale de mise en charge sur la survie et la croissance des larves d'Heterobranchus longifilis (Valenciennes, 1840) élevées en bassins fertilisés
}

\author{
Hyppolite AGADJIHOUEDE ${ }^{1,2 *}$, Antoine CHIKOU ${ }^{1}$, Elie Montchowui ${ }^{1}{ }^{2}$ et Philippe LALEYE 1 \\ 'Laboratoire d'Hydrobiologie et d'Aquaculture / Faculté des Sciences Agronomiques/Université d'Abomey-Calavi, 01 BP \\ 526 Cotonou, Bénin. \\ 2École Nationale supérieure des Sciences et Techniques Agronomiques de Kétou / Université d'Agriculture de Kétou, BP \\ 95 Kétou, Bénin \\ Adresse des auteurs * Auteur correspondant : AGADJIHOUEDE Hyppolite, Tél : (00229) 97265704 ; courriel : \\ agadjihouede@gmail.com \\ CHIKOU Antoine : (00229) 96691353 ; courriel : chikoua@yahoo.fr \\ Montchowui Elie : (00229) 96742994 ; courriel : e.montchowui@yahoo.fr \\ LALEYE Philippe : (00229) 97910784 ; courriel : phlaleye@yahoo.fr
}

Original submitted in on 26 th August 2014. Published online at www.m.elewa.org on 29th December 2014.

http://dx.doi.org/10.4314/jab.v84i1.8

\section{RÉSUMÉ}

Objectif : La présente étude a consisté à évaluer l'effet de la densité initiale de mise en charge sur la survie et la croissance des larves d'Heterobranchus longifilis (Valenciennes, 1840) en bassins fertilisés.

Méthodologie et résultats : L'expérience a été conduite en un replicat dans 08 bassins en béton remplis avec 200 litres d'eau et fertilisés avec de la fiente de volaille sèche (dose : $600 \mathrm{~g} / \mathrm{m}^{3}$ ), puis ensemencés avec $83 \pm 13$ ind/L de zooplancton. Six jours après l'ensemencement du zooplancton, les larves de $H$. longifilis âgées de 2 jours (Pmi $=2,8 \pm 0,1 \mathrm{mg}$ ) ont été directement introduites dans les bassins. La mise en charge est réalisée avec 4 densités (D300 $=300$ individus $/ \mathrm{m}^{3}, \mathrm{D} 400=400$ individus $/ \mathrm{m}^{3}, \mathrm{D} 500=500$ individus $/ \mathrm{m}^{3}$ et D600 $=600$ individus $/ \mathrm{m}^{3}$ ). Ces larves se sont servies exclusivement du zooplancton pendant les 7 premiers jours d'élevage. A partir du 8ème jour, elles sont nourries à l'aliment Coppens jusqu'à 30 jours d'âge. A la fin de l'expérience, la densité D300 a donné les meilleurs taux de survie $(58,33 \pm 0,52 \% ; p<0,05)$ et de conversion alimentaire $(0,28 \pm 0,02 ; p<0,05)$. Les poids moyens finaux sont élevés à toutes les densités et ne sont pas significativement différents. Par ailleurs, les meilleurs taux de croissance spécifique sont obtenus aux densités D300, D500 et D600.

Conclusion et application des résultats : La densité de 300 individus $/ \mathrm{m}^{3}$ constitue la meilleure densité initiale de mise en charge des larves qui donne de meilleures performances de survie et de croissance pour les larves en bassins fertilisés. Ainsi, avec cette densité de mise en charge, ce système offre de réelles perspectives moins onéreuses de production d'alevins de $H$. Iongifilis en milieu paysan.

Mots clés : Heterobranchus longifilis, bassins fertilisés, densité initiale, survie, croissance. 
Agadjihouede et al. J. Appl. Biosci. Effet de densité initiale de mise en charge sur la survie et la croissance des larves d'Heterobranchus longifilis (Valenciennes, 1840) élevées en bassins fertilisés

Effect of initial stocking density on survival and growth of Heterobranchus longifilis(Valenciennes, on 1840) larvae breeding in fertilized ponds

\section{ABSTRACT}

Objective: This study aims to evaluate effect of initial stocking density on the survey and growth of Heterobranchus longifilis larvae breeding in fertilized ponds.

Methodology and results: Experiment was took place in replicate in 8 ponds. Each pond was filled using 200 liters of water and fertilized with dry poultry droppings (doses: $600 \mathrm{~g}$ dry weight $/ \mathrm{m}^{3}$ ). Then the zooplankton was inoculated (density $=83 \pm 13$ ind $/ L$ ). Six days after inoculation of zooplankton, larvae of $H$. longifilis of 2 days after post-hatching (Initial mean weight $=2.8 \pm 0.1 \mathrm{mg}$ ) were directly introduced into ponds. Four initial stocking densities were applied (D300 $=300$ individuals $/ \mathrm{m}^{3}, \mathrm{D} 400=400$ individual $/ \mathrm{m}^{3}, \mathrm{D} 500=500$ individual $/ \mathrm{m}^{3}$ and $\mathrm{D} 600=600$ individual $\left./ \mathrm{m}^{3}\right)$. These larvae used exclusively zooplankton during the first 7 days of breeding. From the 8th day, larvae were fed with Coppens feed until 30 days with age. In the end, D300 gave the best rates of survival ( $58.33 \pm 0.52 \% ; p<0.05)$ and of food conversion $(0.28 \pm 0.02 ; p<0.05)$. Final mean weights were brought up to all the densities and were not significantly different. Furthermore, the best rates of specific Growth were obtained with D300, D500 and D600.

Conclusion and application of findings: The density of 300 individual / $\mathrm{m}^{3}$ constitutes the best initial stocking density of $\mathrm{H}$. longifilis larvae of 2 days after hatching (Initial mean weight $=2.8 \pm 0.1 \mathrm{mg}$ ) which gives better performances of survival and growth of larvae in fertilized ponds. Then, this system offers the cheaper perspectives of production of $H$. longiflis in farmers with this initial stocking density.

Key words: Heterobranchus longifilis, fertilized ponds, initial density, survival, growth.

\section{INTRODUCTION}

L'objectif de cette expérience est de tester la technique d'élevage larvaire en bassins fertilisés sur la production d'alevins de silure africain Heterobranchus longifilis (Valenciennes, 1840) (Figure 1). Des études réalisées précédemment sur l'élevage larvaire des poissons en aquarium avec de proies vivantes (Awaïss et Kestemont, 1992 ; Fiogbé et al., 2003 ; Hounsou, 2006 ; Agadjihouèdé et al., 2012) ont prouvé l'efficacité du zooplancton locale sur les performances de survie et de croissance des larves. Mais l'élevage en aquarium reste encore minuscule et plus fin à être appliquée en milieu rural. Ainsi des essais sont entrepris pour approcher les conditions rurales afin de promouvoir la production d'alevins de cette espèce dans les fermes piscicoles rurales. Par ailleurs, la densité de stockage est un important facteur de production piscicole surtout dans un l'élevage commercial. Elle a été déjà étudiée pour plusieurs espèces piscicoles et varie selon les espèces et le système d'élevage (Boyd et Tucker, 1998; Danaher et al., 2007). A notre connaissance, aucune étude de densité de stockage n'a été réellement réalisée pour l'espèce $H$. longifilis. Or, ce silure africain offre un grand intérêt aquacole. En effet, très robuste, il présente une croissance très rapide et une chair de qualités gustatives très bien appréciées par les consommateurs africains. Selon Hecht et Pienaar (1993), une densité de stockage incorrecte génère des compétitions intraspécifiques, qui résultent d'une ségrégation de l'augmentation de taille et de cannibalisme. La présente étude, a pour but de tester avec les proies naturelles locales, l'élevage des larves de $H$. longifilis en bassins fertilisés et de déterminer la densité initiale de mise en charge qui minimise l'hétérogénéité des tailles des larves tout en améliorant leur croissance et leur survie. 


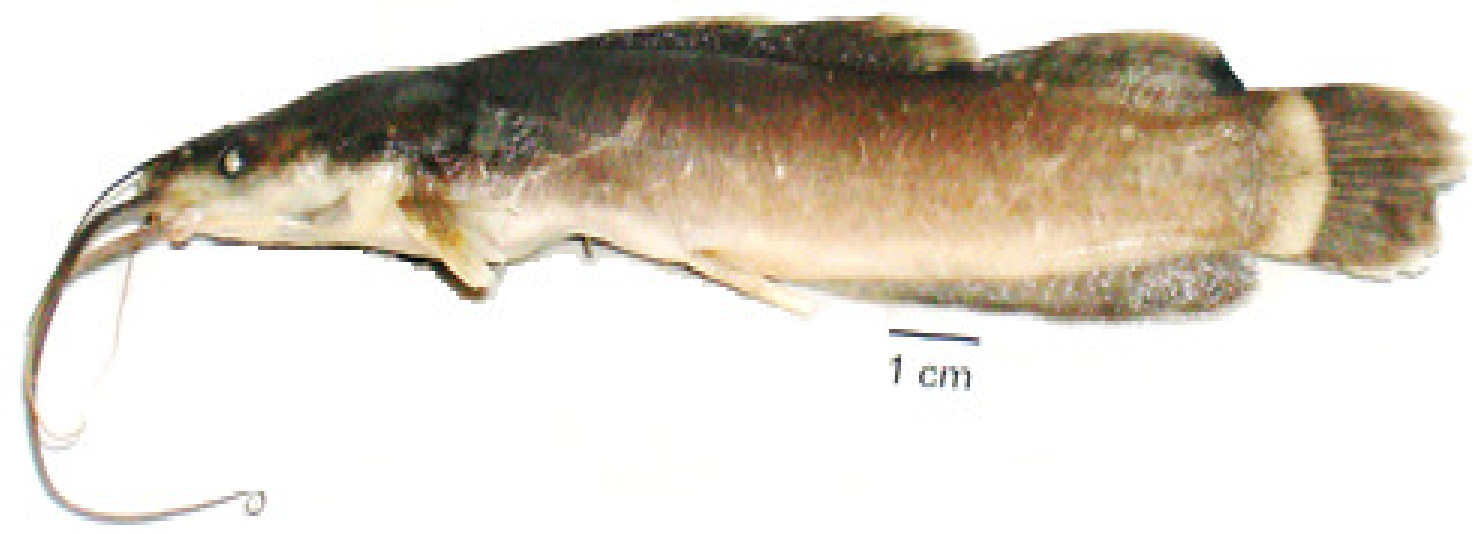

Figure 1 : Heterobranchus longifilis (Valenciennes, 1840). Source : Cliché AGADJIHOUEDE, 2014

\section{MATÉRIEL ET MÉTHODES}

Origine des larves : Les larves de $H$. longifilis utilisées étaient issues de la reproduction artificielle des géniteurs et a été réalisée dans les locaux de l'Unité de Recherche et de la Formation en Pisciculture (URFP) de l'Université d'Abomey-Calavi.

La production de larves de cette espèce nécessite un certain nombre de manipulations dont la chronologie est la suivante :

- Induction de la ponte par injection d'extraits de l'ovaprim SYNDEL International Inc. Canada (info@syndel.com) et mise en stabulation des femelles à une température de $\pm 25^{\circ} \mathrm{C}$.

- L'extraction des ovules (Stripping), fécondation des œufs par laitance de testicules et incubation des œufs dans des paniers placés dans les bacs d'incubation (température supérieure à $25^{\circ} \mathrm{C}$ ).

- Éclosion des œufs, retrait des paniers et évacuation des œufs non éclos.

- Résorption de la vésicule vitelline.

\section{Installation expérimentale}

Préparation des bassins : 4 bassins de $1 \mathrm{~m} \times 1 \mathrm{~m} \times 1 \mathrm{~m}$ chacun sont utilisés pour l'essai. Deux jours avant le début de l'étude, les bassins ont été soigneusement lavés à l'eau de javel, rincés abondamment et laissés séchés pour les débarrasser de toute impureté. Ces bassins sont remplis avec 200 litres d'eau (eau de consommation locale (4/5) + eau d'étangs filtrés (1/5)) et fertilisés avec de la fiente de volaille sèche à raison de $600 \mathrm{~g} / \mathrm{m}^{3}$ d'eau
(Agadjihouèdé et al., 2011). Chaque jour, le niveau d'eau était mesuré au moyen d'une échelle graduée posée dans un coin du bassin. Au besoin, de l'eau était rajoutée afin de maintenir l'eau au niveau initial. Deux jours après la fertilisation, un concentré de $100 \mathrm{ml}$ de zooplancton est ensemencé dans chaque bassin soit une densité de $83 \pm$ $15 \mathrm{ind} / \mathrm{L}$. Cette production de zooplancton est lancée dans les bassins 6 jours avant la mise en charge des larves.

Mise en charge des larves : La mise en charge des larves a eu lieu 6 jours après l'ensemencement au zooplancton. Deux jours après éclosion, les larves de $H$. longifilis ont été comptées et introduites directement dans les bassins. La mise en charge est réalisée avec 4 densités à savoir D300, D400, D500 et D600 correspondant respectivement à $300,400,500$ et 600 individus $/ \mathrm{m}^{3}$. Un échantillon de 200 larves a été pesé ; ce qui donne un poids moyen individuel de 2,8 $\pm 0,1 \mathrm{mg}$. Ces larves se sont servies exclusivement du zooplancton pendant 7 jours soit $\mathrm{J} 9$ post-éclosion. A partir du gème jour post-éclosion, ces alevins sont sevrés à l'aliment sec et sont ainsi nourris au Coppens. Les compositions de l'aliment Coppens utilisé sont présentées dans le tableau 1. L'élevage a duré 28 jours, soit de $2 \mathrm{j}$ à $30 \mathrm{j}$ postéclosion. Le nourrissage s'est fait d'abord au taux de $10 \%$ de la biomasse, puis $9 \%$ et enfin $8 \%$ (chaque fois pendant 7 jours) jusqu'à la fin de l'essai (Rukéra Tabaro et al., 2005). L'essai a été répliqué une fois.

Tableau 1 : Composition biochimique de l'aliment du Coppens 
Agadjihouede et al. J. Appl. Biosci. Effet de densité initiale de mise en charge sur la survie et la croissance des larves d'Heterobranchus longifilis (Valenciennes, 1840) élevées en bassins fertilisés

Table 1 : Biochemical Composition of Coppens

\begin{tabular}{|l|c|}
\hline \multicolumn{1}{|c|}{ Composition } & Quantité \\
\hline Protéines brutes $(\%)$ & 45 \\
\hline Lipides bruts $(\%)$ & 10,4 \\
\hline Fibres $(\%)$ & 1,3 \\
\hline Cendres $(\%)$ & 7,3 \\
\hline Cadnium $(\%)$ & 0,7 \\
\hline Phosphore $(\%)$ & 1,3 \\
\hline Vitamine $\mathrm{E}(\mathrm{mg} / \mathrm{kg})$ & 200 \\
\hline Vitamine C $(\mathrm{mg} / \mathrm{kg})$ & 300 \\
\hline Taille $(\mu \mathrm{m})$ & $200-300$ \\
\hline
\end{tabular}

Source : Coppens International (2005)

Contrôle de la qualité de l'eau : Il a consisté à la prise des paramètres physico-chimiques $(\mathrm{pH}$, température et oxygène dissous) dans les bassins. lls ont été relevés 3 fois par jour ( $7 \mathrm{~h}, 12 \mathrm{~h}$ et $17 \mathrm{~h}$ ) et ceci tous les jours à l'exception des jours de contrôle de croissance.

Contrôle de croissance des larves : Le contrôle de croissance des larves a été réalisé tous les 7 jours sur des échantillons de $2 / 3$ des individus prélevés au hasard dans chaque bassin. Ces individus ont été individuellement pesés sur une microbalance de marque SARTORIUS (précision $=0,1 \mathrm{mg}$ ) après les avoir passés rapidement sur du papier mouchoir pour enlever l'eau sur leur corps.

Parallèlement, un comptage systématique de tous les individus a été effectué en vue d'évaluer la survie des larves.

Traitement des données : Les données collectées sont utilisées pour le calcul de la survie et des paramètres de croissance.

* La survie (S) des larves a été estimée sur la base des moyennes mobiles de leur effectif au cours du suivi. Elle est exprimée en pourcentage de l'effectif initial (nombre de larves d'âge 2 jours mises en charge dans les bassins) en fonction de l'âge des larves.

$S(a)=100 \times N(a) / N(2)$

où, $S$ = pourcentage de survie ; $a$ = âge ; $N$ = effectif.

\section{RÉSULTATS}

Caractéristiques du milieu d'élevage : L'évolution de la température au cours de l'expérience n'a pas marqué beaucoup de fluctuations. La moyenne des températures de tous les bassins a été de $28,2 \pm 1,5^{\circ} \mathrm{C}$, avec de plus faibles températures observées le matin et de plus hautes en fin d'après-midi. Les valeurs extrêmes ont été de 26,6 ${ }^{\circ} \mathrm{C}$ et $31,5^{\circ} \mathrm{C}$. Les concentrations moyennes journalières
* Les différents paramètres de croissance ont été calculés de la façon suivante :

-SGR $=100\left(\operatorname{LnP}_{2}-\operatorname{LnP}_{1}\right) \cdot \Delta t^{-1}$

SGR = taux de croissance spécifique $\left(\% \mathrm{j}^{-1}\right)$

$\mathrm{LnP}_{1}=$ logarithme népérien du poids initial

$\mathrm{LnP}_{2}=$ logarithme népérien du poids final

$P_{1}$ et $P_{2}=$ poids moyens initial et final des poissons $(\mathrm{mg})$

- $\mathrm{TCA}=\mathrm{PA}\left(\mathrm{Bm}_{2}-\mathrm{Bm}_{1}\right)^{-1}$

TCA $=$ taux de conversion alimentaire

$\mathrm{Bm}_{1}$ et $\mathrm{Bm}_{2}=$ biomasses initiale et finale des poissons par bassin $(\mathrm{mg})$

$\mathrm{PA}=$ poids sec d'aliment distribué par bassin ( $\mathrm{mg}$ ).

Les résultats sont présentés sous forme de moyennes \pm erreur-type entre replicat. La comparaison des taux de survie a été réalisée en utilisant le test de $\chi^{2}$ et celle des poids moyens finaux et des taux de croissance spécifique et de conversion alimentaire a été réalisée en procédant à une analyse de variance (ANOVA). Lorsque ces tests révélaient une différence significative, des comparaisons "Post Hoc" (Least Significant Difference : LSD) ont été exécutées. Dans tous ces tests statistiques, les différences ont été considérées significatives au seuil de $5 \%$. Les analyses ont été effectuées à l'aide du programme Statview (Version 6, SAS Institute Inc.).

d'oxygène dissous dans tous les bassins ont varié autour de 6,6 $\pm 1,2 \mathrm{mg} \cdot \mathrm{L}^{-1}$. Les concentrations extrêmes enregistrées ont été de 5,5 et de $13 \mathrm{mg} . \mathrm{L}^{-1}$. Les variations journalières de l'oxygène dissous étaient relativement élevées, avec des valeurs maximales notées vers $12 \mathrm{~h}$ $13 \mathrm{~h}$ et des valeurs minimales en fin de journée. Cependant, ces valeurs minimales étaient largement 
supérieures à 0 , ce qui évitait des anoxies temporaires comme il en existe souvent dans les petits bassins d'eau tropicale eutrophe, et dans les étangs de pisciculture très enrichis. La valeur moyenne du $\mathrm{pH}$ de l'eau dans les bassins est de 7,7 $\pm 0,2$. Les valeurs minimale et maximale sont comprises entre 7,2 et 8,6 . Dans l'ensemble, aucune valeur extrême, léthale ou limitante, basique ou acide, n'est observée au cours du cycle d'étude.

Survie et croissance des larves : Les différents paramètres de survie et de croissance des larves sont consignés dans le tableau 2

Tableau 2 : Paramètres de survie et de croissance de Heterobranchus longifilis après 28 jours d'élevage en bassins fertilisés avec la fiente de volaille.

Table 2: Parameters of survival and growth of Heterobranchus longifilis after 28 days of breeding in ponds fertilized with poultry droppings.

\begin{tabular}{lccccc}
\hline & D300 & D400 & D500 & D600 & p \\
\hline Pmi (mg) & $2,8 \pm 0,1$ & $2,8 \pm 0,1$ & $2,8 \pm 0,1$ & $2,8 \pm 0,1$ & $>0,05$ \\
Pmf (mg) & $3228,39 \pm 0,11$ & $3055,72 \pm 0,16$ & $3643,61 \pm 0,17$ & $3691,01 \pm 0,32$ & $>0,05$ \\
CV final (\%) & $22,65 \pm 0,52^{\mathrm{a}}$ & $21,03 \pm 0,85^{\mathrm{a}}$ & $33,51 \pm 0,56^{\mathrm{b}}$ & $32,97 \pm 0,5^{\mathrm{b}}$ & $<0,05$ \\
SGR (\%.J-1) & $25,20 \pm 0,31^{\mathrm{ab}}$ & $24,98 \pm 0,14^{\mathrm{a}}$ & $25,61 \pm 0,12^{\mathrm{b}}$ & $25,65 \pm 0,26^{\mathrm{b}}$ & $<0,05$ \\
TCA & $0,28 \pm 0,02^{\mathrm{a}}$ & $0,53 \pm 0,27^{\mathrm{b}}$ & $0,45 \pm 0,9^{\mathrm{b}}$ & $0,44 \pm 0,13^{\mathrm{b}}$ & $<0,05$ \\
Survie finale (\%) & $58,33 \pm 0,52^{\mathrm{a}}$ & $37,5 \pm 0,52^{\mathrm{b}}$ & $47 \pm 0,85^{\mathrm{c}}$ & $10,83 \pm 0,71^{\mathrm{d}}$ & $<0,05$ \\
\hline
\end{tabular}

(Pmi = poids moyen initial, $\mathrm{Pmf}=$ poids moyen final, $\mathrm{Cv}=$ coefficient de variation du poids, $\mathrm{SGR}=$ taux de croissance spécifique, et $\mathrm{TCA}=$ taux de conversion alimentaire). Pour chaque ligne, les valeurs portant des lettres différentes sont significativement différentes (Post Hoc: LSD, $p<0,05$ ); les erreurs-types ont été calculés entre replicats.

$(\mathrm{Pmi}=$ initial mean weight, $\mathrm{Pmf}=$ final mean weight, $\mathrm{Cv}=$ Coefficient of variation of the weight, $\mathrm{SGR}=$ Specific growth rate, and TCA = conversion rate of food. Values followed by the same superscript are not significantly different $(p>0.05)$ from others in the same line; standard deviations were calculated among replicate.

* Survie : Les variations du pourcentage de survie en fonction de l'âge des larves sont présentées dans la figure 2. Les plus fortes survies des larves de 9 jours d'âge sont observées dans les bassins à D300 avec un pourcentage de survie de $83,33 \pm 0,00 \%$. Dans les bassins à D400, la survie est respectivement $65 \pm 0,73 \%$ à ces âges-là ; tandis que dans les bassins à D500 et $D 600$, les pourcentages de survie sont de $52 \pm 0,49$ et de $35 \pm 0,64 \%$. La survie des larves diminue ensuite progressivement dans tous les bassins pour atteindre aux 23 ème jours d'âge des taux de 58,33 \pm 0,52 \%, 37,5 \pm
$0,52 \%, 47,00 \pm 0,85 \%$ et $10,83 \pm 0,71 \%$ respectivement dans les bassins à D300, D400, D500 et D600. A partir de ce moment, elle se maintient constante dans tous les bassins jusqu'à la fin de l'expérience. La comparaison de ces taux de survie des larves obtenus à la fin de l'expérience par le test de $X^{2}$ a montré une différence significative $(p<0,05)$ entre toutes les densités à l'exception des densités D400 et D500 où les taux de survie ne révèlent aucune différence significative entre eux $(p>0,05)$. 


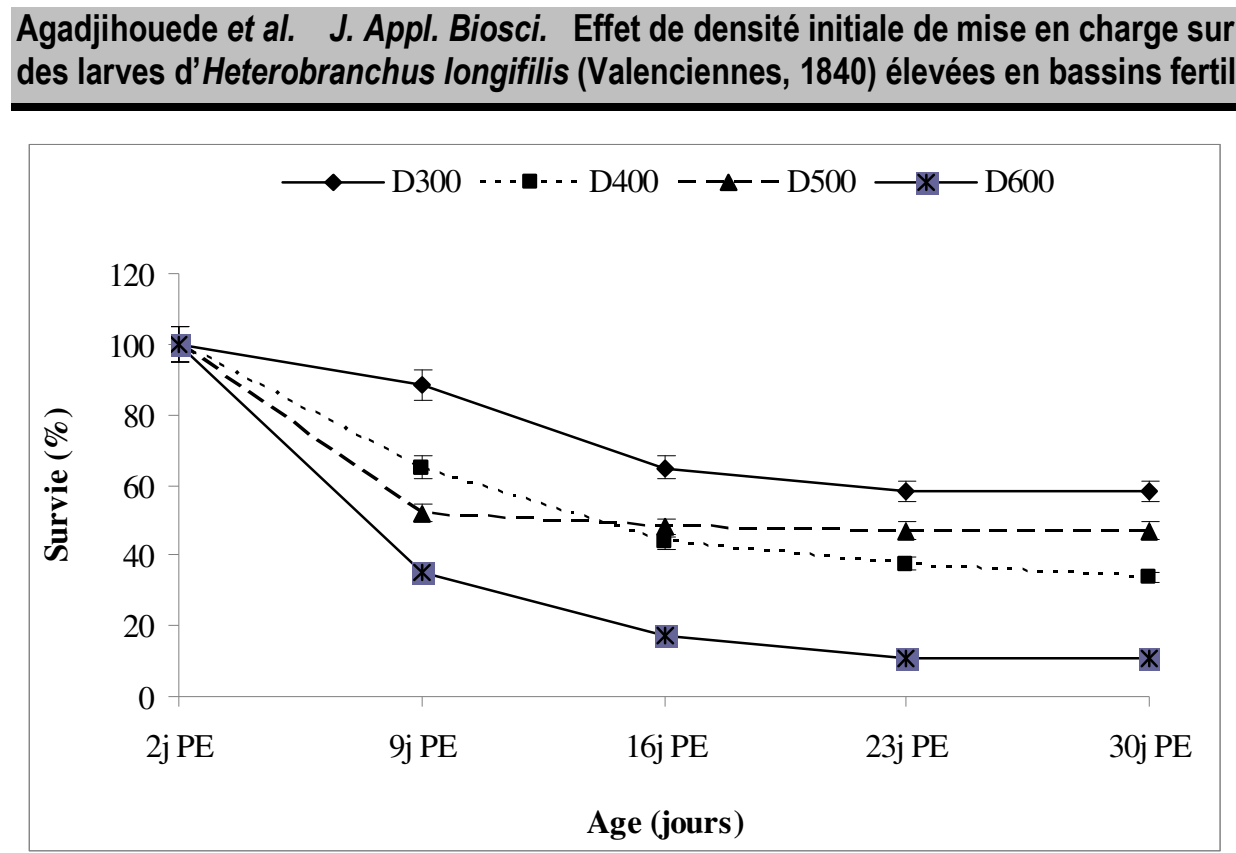

Figure 2 : Évolution de la survie des larves en fonction de leur âge dans les bassins

Fig. 2: Survival evolution of larvae according to their age in ponds

2j PE, 9j PE, ... 30j PE = 2 jours Post-éclosion, 9 jours Post-éclosion, ..., 30 jours Post-éclosion.

Les barres verticales représentent les écarts entre réplicats

* Croissance: Après les 28 jours d'élevage, aucune différence significative (Post Hoc: LSD, $p>0.05$ ) n'a été observée entre les poids moyens finaux obtenus aux densités D300, D400, D500 et D600 (figure 3). Les poids moyens finaux les plus élevés ont été enregistrés à la densité $\mathrm{D} 600(3691,01 \pm 1,21 \mathrm{mg})$ et les plus faibles à la densité D400 (3055,72 $\pm 0,63 \mathrm{mg})$. Les valeurs de $\mathrm{Cv}$ des poids moyens finaux ont évolué dans le même sens. Aux densités D300 et D400 et aux densités D500 et D600, aucune différence significative n'a été notée entre ces valeurs de Cv (Post Hoc: LSD, $p>0.05$ ) ; par contre, la différence a été significative entre ces densités prises deux à deux (Post Hoc: LSD, $p<0.05$ ) (figure 3). Les meilleurs taux de croissance spécifique (SGR) des larves ont été obtenus à la densité $\mathrm{D} 600(25,66 \pm 0,26 \%$.J-1) . Ces taux ne sont pas significativement différents de ceux enregistrés aux densités D300 et D500 ; mais ils sont significativement différents de ceux obtenus à la densité D400. Les valeurs de TCA obtenues à ces densités sont différentes (Post Hoc: LSD, $p<0.05$ ) les unes des autres: les valeurs les plus intéressantes ont été enregistrées à la densité $\mathrm{D} 300(0,28 \pm 0,003)$ et les moins intéressantes à celle de D400 $(0,53 \pm 0,3)$. 

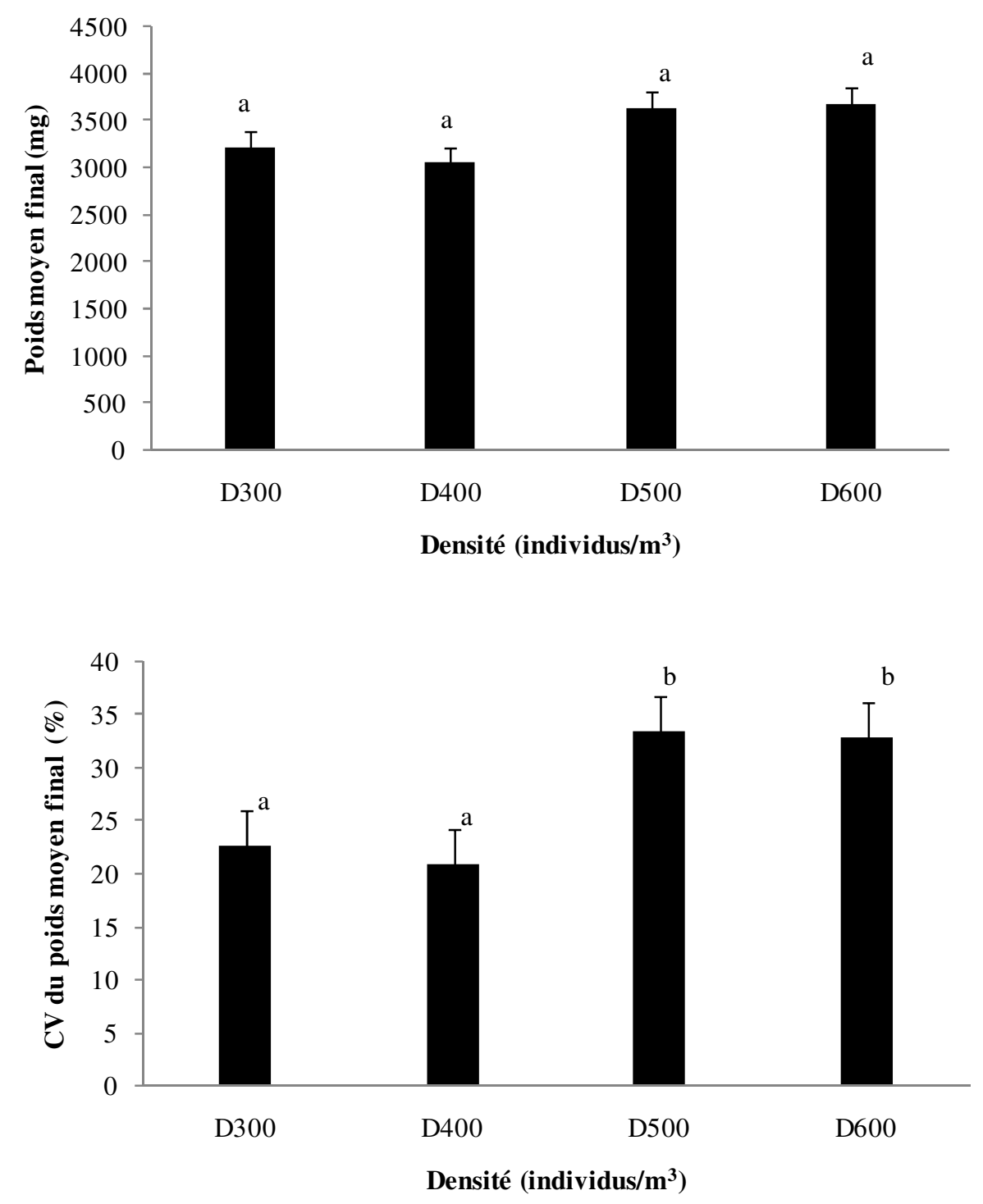


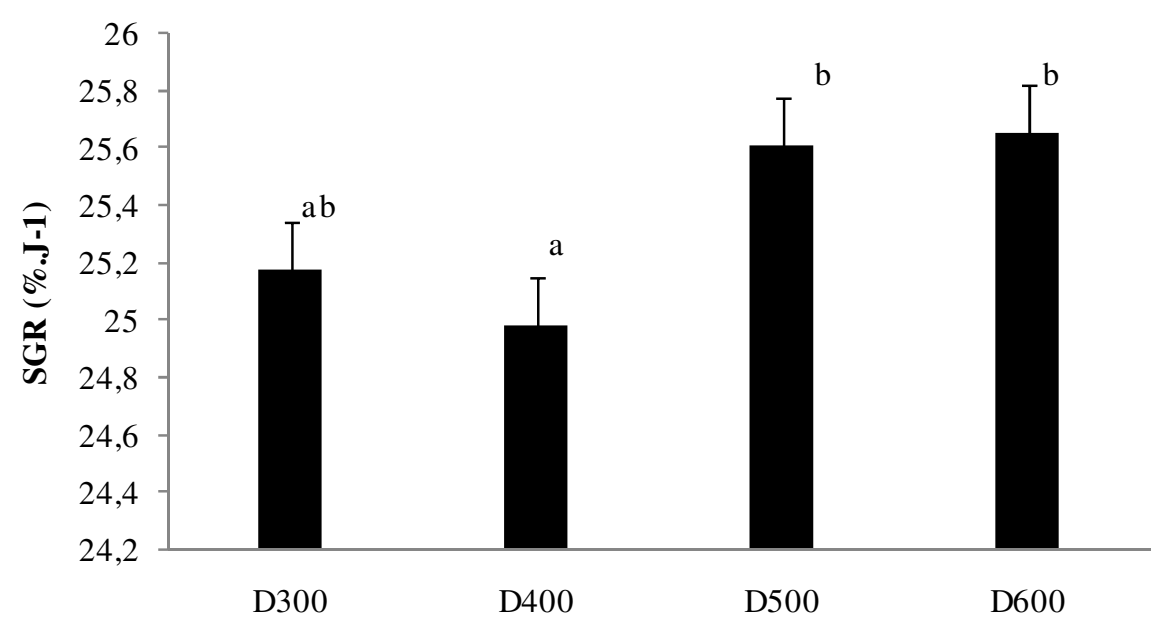

Densité (individus $/ \mathbf{m}^{3}$ )

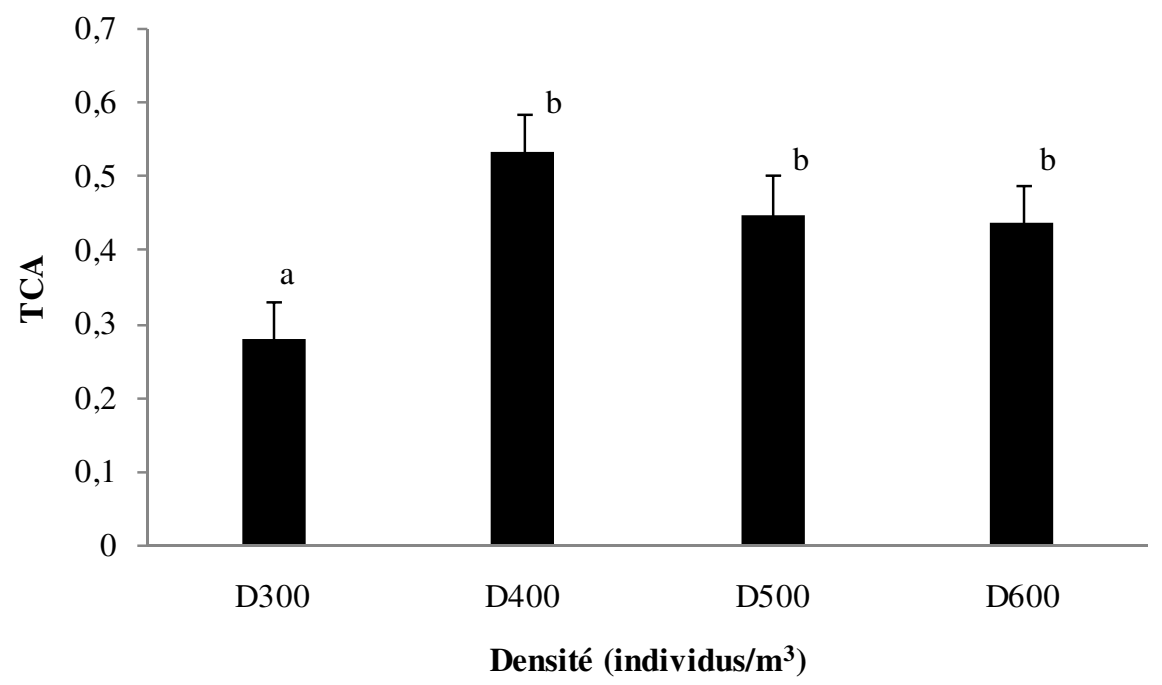

Figure 3 : Effet de densité de mise en charge sur les paramètres de croissance des larves de $H$. longifilis élevées en bassins fertilisés avec la fiente de volaille pendant 28 jours.

Fig. 3: Effect of stocking density on the parameters of growth of $\mathrm{H}$. Iongifilis larvae breeding in ponds fertilized with poultry droppings during 28 days.

Pour chaque paramètre, des lettres différentes au dessus des histogrammes indiquent une différence significative $p<0,05$ (Post Hoc: LSD). Les barres verticales représentent les écarts entre réplicats $n=2$.

\section{DISCUSSION ET CONCLUSION}

Les paramètres physico-chimiques de l'eau des bassins sont restés conformes aux normes recommandées pour la survie et la croissance de $H$. Iongifilis en élevage qui sont de $25-33^{\circ} \mathrm{C}$ pour la température de l'eau et de 6,58,5 pour le pH (Legendre et Teugels, 1991 ; Luquet et al., 1993 ; Gilles et al., 2001). La densité D300 (300 individus/ $\mathrm{m}^{3}$ ) présente la meilleure survie des larves. Les taux de survie enregistrés tout au long de l'élevage avec cette densité D300 sont significativement plus élevés que ceux enregistrés avec toutes les autres densités (D400, D500 et $\mathrm{D600)}$. Ceci pourrait s'expliquer sans doute par le fait que les larves des densités D400, D500 et D600 beaucoup plus nombreuses devaient se partager un stock de nourriture qui est à peu près identique à celui de la densité D300. La survie de $83,33 \pm 0,00 \%$ obtenue avec cette densité D300 après les 7 jours d'élevage (nourrissage exclusif des larves au zooplancton) soit 9 jours d'âge est élevée et nettement supérieure à celles de 


\section{Agadjihouede et al. J. Appl. Biosci. Effet de densité initiale de mise en charge sur la survie et la croissance}

des larves d'Heterobranchus longifilis (Valenciennes, 1840) élevées en bassins fertilisés

40 - 50\% notée par Barro (1995) pour la même espèce en 11 jours. Elle est comparable à la survie de $82-99 \%$ enregistrée par Otémé et Gilles (1995) après 10 jours d'élevage avec des nauplii d'Artemia. Cette survie baisse ensuite pour donner en fin d'expérience des taux relativement faibles (moyenne $=58,33 \pm 0,52 \%$, tableau 2) en comparaison avec ceux notés par Imorou Toko et al. (2003). Cette grande mortalité en fin d'expérience est due au cannibalisme (Baras et al., 1999) exercé par des jumpers péchés à partir de 16 ème jours, signe qu'ils commencent à être abondants. En effet, il est bien connu que chez certains poissons-chats, la mortalité due à ce comportement est liée à la différence de tailles au sein de la population (Hecht \& Appelbaum, 1988; Appelbaum \& Kamler, 2000). L'estimation du risque de cannibalisme au sein d'une population a été abordée dans plusieurs travaux (Appelbaum \& Kamler, 2000 ; Baras \& D'Almeida, 2001; Brown \& Braithwaite, 2004). Ce risque est d'autant plus grand que la variabilité de taille est élevée (Appelbaum \& Kamler, 2000). Dans la présente étude, cette variabilité a été estimée au moyen du coefficient de variation du poids. Ainsi les plus faibles valeurs de ce coefficient de variation enregistré aux densités D300 et D400 montrent que les individus de ces populations sont de taille plus homogène par rapport aux individus des densités D500 et D600. Ceci justifierait également les meilleures survies obtenues aux densités D300. Par ailleurs, avec la densité D300, la croissance des larves est aussi bonne. Les poids moyens finaux sont élevés et ne sont pas significativement différents quelle que soit la densité. Au bout des 28 jours d'élevage, les poids moyens des larves se sont accrus énormément en passant de 2,8 $\pm 0,1 \mathrm{mg}$ à $3691,01 \pm 0,32 \mathrm{mg}$ (poids

\section{RÉFÉRENCES}

Agadjihouèdé $\mathrm{H}$, Chikou $\mathrm{A}$, Bonou $\mathrm{CA}$, Lalèyè $\mathrm{Ph}, 2012$. Survival and growth of Clarias gariepinus and Heterobranchus longifilis larvae fed with freshwater zooplankton. Journal of Agricultural Science and Technology B2: 192-197.

Agadjihouèdé $\mathrm{H}$, Bonou $\mathrm{CA}$, Montchowui $\mathrm{E}$, Lalèyè $\mathrm{Ph}$, 2011. Recherche de la dose optimale de fiente de volaille pour la production spécifique de zooplancton à des fins piscicoles. Cahiers Agricultures 20(4): 247-260.

Appelbaum S, Kamler E, 2000. Survival, growth, metabolism and behaviour of Clarias gariepinus (Burchell, 1822) early stages under different light conditions. Aquaculture England 22: 269-287.

Awaïss A, Kestemont K, Micha J-C, 1992. Nutritional suitability of the Rotifer Brachionus calyciflorus maximum obtenu avec la D600). Ce qui montre que le système adopté fonctionne correctement. Les taux d'accroissement obtenus sont élevés avec toutes les densités; les meilleurs taux étant obtenus avec les densités D600, D500 et D300 qui ne sont pas significativement différents. De plus, la densité D300 donne le meilleur taux de conversion alimentaire comparativement aux autres densités. Ces taux de conversion alimentaires obtenues au cours de cette expérience indiquent de bonnes performances au regard des résultats obtenus par Luquet (1996) chez $H$. longifilis et Imorou Toko et al. (2003) chez C. gariepinus.

D'une façon générale, le système d'élevage proposé s'est avéré très efficace pour l'amélioration des performances de croissance des larves de $\mathrm{H}$. longifilis de 2 jours (en fin de résorption vitelline) quelle que soit la densité de mise en charge $\left(300,400,500\right.$ et 600 individus $\left./ \mathrm{m}^{3}\right)$. Par contre, comparé à d'autres systèmes, notamment les travaux de Legendre et Teugels (1991) où les chercheurs ont obtenu une survie moyenne de $68 \%$ avec des nauplii d'Artemia salina comme aliment de départ, le présent système paraît moins efficace en terme de survie. Toutefois, avec la densité D300, les performances de survie appréciable et de croissance élevée obtenues montrent que le système est applicable en milieu paysan. En conclusion, cette expérience a montré que le système d'élevage des larves en bassins fertilisés avec la fiente de volaille proposé dans la présente étude est réalisable. La densité qui permet d'obtenir de bon taux de survie et de bonne croissance des larves est la D300. Ainsi, avec une densité de mise en charge de 300 individus $/ \mathrm{m}^{3}$, ce système offre de réelles perspectives moins onéreuses de production d'alevins de $H$. longifilis en milieu paysan.

Pallas for rearing freshwater fish larvae. Journal of Applied Ichtyology 8:263-270.

Baras E, D'Almeida AF, 2001. Size heterogeneity prevails over kinship in shaping cannibalism among larvae of sharptooth catfish Clarias gariepinus. Aquatic Living Resources 14: 251-256.

Baras E, Tissier F, Philipart JC, Mélard C, 1999. Sibling cannibalism among juvenile vundu under controlled conditions. II. Effect of body weight and environmental variables on the periodicity and intensity of type II cannibalism. Journal of Fisheries and Biology 54: $106-118$.

Barro M, 1995. Étude de la dynamique et de l'efficience d'un système d'élevage mixte de larves d'Heterobranchus longifilis et de proies dominées par les rotifères (Station d'aquaculture 
d'Anna, Côte d'Ivoire, Afrique. Mémoire présenté à l'université du Québec à Chicoutimi : $86 \mathrm{p}$.

Boyd CE, Tucker CS, 1998. Pond aquaculture water quality management. Kluwer Academic Publishers, Norwell, Massachusetts, USA.

Brown C, Braithwaite V, 2004. Size matters : a test of boldness in eight populations of the poeciliid Brachyraphis episcope. Animal Behaviors 68: 1325-1329.

Danaher JJ, Tidwell JH, Coyle SD, Dasgupta S, 2007. Effects of Two Densities of Caged Monosex Nile Tilapia, Oreochromis niloticus, on Water Quality, Phytoplankton Populations, and Production When Polycultured with Macrobrachium rosenbergii in Temperate Ponds. Journal of World Aquaculture Society 38 (4): 367-382.

Fiogbé ED, Kestemont P, Micha J-C, 2003. Performances zootechniques comparées de Rotifères d'eau douce Brachionus calyciflorus et de nauplii d'Artemia chez les larves de la perche fluviatile Perca fluviatilis. Tropicultura 21(1) :3135.

Gilles S, Dugué R, Slembrouk J, 2001. Manuel de production du silure africain Heterobranchus longifilis. Maisonneuve et Larose, Paris, $128 \mathrm{p}$.

Hecht T, Appelbaum A, 1988. The culture of sharptooth catfish Clarias gariepinus in Southern Africa. South African Journal of Sciences 153: 121-133.

Hecht T, Pienaar AG, 1993. A review of cannibalism and its implications in fish larviculture. Journal of World Aquaculture Society 24: 246-261.

Hounsoun L.C., 2006. Étude comparée de la survie, de la croissance et du coût de production des larves de Clarias gariepinus (Burchell, 1822), nourries aux larves de même espèce, aux rotifères et à l'aliment sec en bassin. Mémoire d'ingénieur agronome, Faculté des Sciences Agronomiques, Université d'Abomey-Calavi : $49 \mathrm{p}$.
Imorou Toko I, Fiogbe ED, Kestemont P, 2003. Essai d'élevage de Clarias gariepinus en whédo. Résumé présenté à la 3ème conférence de l'association Panafricaine des Pêches (PAFFA) ; Cotonou du 10 au 14 /11/2003.

Legendre M, Teugels GG, 1991. Développement et tolérance à la température des œufs de Heterobranchus longifilis, et comparaison des développements larvaires de $H$. longifilis et de $C$. gariepinus (Teleostei, Clariidae). Aquatic Living Resources 4: 227-240.

Luquet $\mathrm{P}, 1996$. Valeur prédictive de la croissance linéaire pour l'établissement de tables de rationnement pour le poisson: cas du silure africain Heterobranchus longifilis. Journées INRA-IFREMER, Nutrition des poissons ; 21-22 fév. $1996 ; 4$ p.

Luquet P, Otémé ZJ, Metongo SB, 1993. Élevage du silure Heterobranchus longifilis Val. en bassin sans renouvellement d'eau : effets de l'évolution de quelques paramètres chimiques de l'eau sur la croissance et la survie. Journal Ivoirien d'Océanologie et de Limnologie 2(2) : 43-53.

Otémé ZJ, Gilles S, 1995. Élevage larvaire du silure africain Heterobranchus longifilis : évaluation quantitative des besoins en proies vivantes des larves. Aquatic Living Resources 8: 351-354.

Otémé ZJ, Hem S, Legendre M, 1996. Nouvelles espèces de poisons-chats pour le développement de la pisciculture africaine. Aquatic Living Resources 9(hors série) : 207217.

Rukéra Tabaro S, Micha J-C, Ducarme C, 2005. Essais d'adaptation de production massive de juvéniles de Clarias gariepinus en conditions rurales. Tropicultura. 23(4) : 231-244. 